\title{
LÍNEAS DE TRABAJO
}

\section{EXPERIENCLA DIDÁCTICA DE CAMPO DE LA SIERRA CALDERONA, COMUNIDAD VALENCIANA}

\section{El grupo de trabajo}

Somos un pequeño grupo de profesionales de la educación y de las Ciencias Biológicas, que formamos un equipo multidisciplinar y que estamos interesados en facilitar el conocimiento sistémico del medio, a través de una metodología activa, para fomentar la conservación de la Naturaleza, basada en una educación más viva y participativa de la Ecología en particular y de las Ciencias Naturales en general,

Hay que ser conscientes de que la humanidad depende de la educación, por lo que la escuela tiene el deber de una ingente y urgente tarea de educación medioam biental, por lo cual el contacto con la Naturaleza es insustituible para dicha educación. Por todo ello, nosotros pretendemos trabajar la Ecología dentro y fuera del aula, para poder plasmar los conocimientos, técnicas, habilidades, aptitudes y actitudes que consigamos con la presente experiencia didáctica.

\section{El itinerario didáctico «Fuente de Ia Umbrias)}

Los programas actuales de las Ciencias de la Naturaleza son parte importante y necesaria de la formación básica del alumno por cuanto proporcionan conocimientos y experiencias que le ayudan a comprender el medio ambiente que le rodea. El alumno debe ser activo y organizar su trabajo para desarrollar su actitud científica: capacidad de observacion, reconocimiento de situaciones, búsqueda de explicaciones y soluciones.

Varios son los recursos didácticos que se ofrecen en la actualidad para alumnos y profesores: medios audiovisuales, laboratorios, museos, aulas de ta naturaleza, itinerarios didácticos, etc. Sin embargo, estos recursos son todavía escasos, especialmente los extraescolares.
El trabajo ha sido elaborado por un equipo multidisciplinar donde han participado pedagogos, profesores de $\mathrm{EGB}$, biólogos e ingenieros técnicos. Partiendo de un terreno donde desarrollar el itinerario, sobre un camino o sendero preexistente, se buscó la zona más idónea en cuanto a situación y características. Posteriormente se investigó el terreno para ver las posibilidades que ofrecia y se estudiaron sus caracter ísticas biologicas. Se elaboró una primera guía del alumno que se experimentó con varios grupos de diferentes niveles desde $5^{\circ}$ a $8^{\circ}$ de EGB, para ir modificándola según las necesidades observadas y las sugerencias de los profesores. Por último se elaboró là Guía del alumno definitiva y está en perspectiva elaborar un libro descriptivo del itinerario y la sierra circundante, que puede servir at profesor como ibro de consu?ta para resolver las cuestiones que pudieran plantear los alumnos.

En cuanto a la descripción general del Itinerario, podemos decir: que parte de una balsa de riego, pasa por cuttivos de regadio y secano, a continuación por zonas de matorral y campos invadidos por él, para llegar a una fuente que hace de punto de reflexión sobre la experiencia didáctica. A partir de aquí se entra en un medio ambiente menos humanizado y más natural, una zona de encinas, el barranco y por último el pinar, para concluir en un mirador que da al valie dcl Palancia, cerca de otra fuente.

\section{Fichero de actividades prácticas}

El contacto obtenido con los profesores gracias al Itinerario Didáctico nos mostró la falta de recursos, apoyos, conocimientos y materiales con los que deberian contar para introducir la Ecología -en su pleno sentido y complejidad - en la escuela. Esto nos llevó a la necesidad de facilitar al profesorado una serie de actividades y de técnicas de trabajo de campo y aula fácilmente realizables.

La idea era aportar un cúmulo de experiencias (adaptadas a los diferentes momentos psicoevolutivos de los alum. nos y que atendieran tanto a la adqujsición de conocimientos como a los aspectos afectivos) que lleven a una nueva concepción de la Ecología y al descubrimiento del medio ambiente de una forma activa y motivadora, atendiendo a las interrelacioncs entre los seres vivos y su entorno.

Creemos que este recurso didáctico puede llenar un vacio en la enseñanza de estas áreas ya que su enfoque va orientado a imbuir a los alumnos y profesores una mentalización de conservación de la Naturaleza y en especial de la Ecologia a la vez que permite establecer en ellos nucvas pautas de comportamiento, nuevas actitudes de comprension de su medio ambiente, su disfrute racional y la resolución de los muchos problemas que le afectan.

\section{Aprovechamiento ecoeducativo de la Sierra de Calderona}

No satisfechos con el ltinerario Didáctico y con el Fichero de Actividades de Ecología (en fase de elaboración) creimos conveniente llevar a la práctica un proyecto de investigación didácticoecológico más amplio que abarcara los dos trabajos anteriores.

Las características del proyecto que en estos momentos queremos realizar están basadas en una acción preventiva que conlleve una concienciación gencralizada en la educación y pueda rescatar para la historia y las futuras generaciones el patrimonio cultural de este lugar geográfico. La tarea de educación ambiental en el presente proyecto va dirigida hacia un trabajo de campo que dé oportunidades a los educandos a estudiar el medio urbano como rural, en sus condiciones actuales.

Catalán, J.P., Martín, A., Portet, J., Soria, J.M., Bosch, A., Bernabeu, R.E., Ribes, M.L., Vicó, E., Lacuesta, V., López, P. y Muñoz, M.J. Grupo de Educación Medioambiental. Servei de Formació Permanent. Universidad de Valencia. 
DIDÁCTICA DE LA ZOOLOGÍA A NVEL DE $6^{\circ}$ DE EGB: IMPORTANCIA DE LOS CONCEPTOS FILOGENÉTICOS EN LA INTERPRETACIÓN DE LA CLASIFICACIÓN DE LOS ANIMALES.

En el transcurso de los últimos años, algunos profesores de Ciencias Naturales que ejercemos en diferentes niveles de la educación básica o superior, nos hemos venido interesando por la realización de experiencias en el campo de la Didáctica de las diferentes ra mas de la Biología, en especial de la Zoología. Fruto de ello fue un estudio realizado sobre la importancia de los conceptos filogenéticos en la interpretación de la clasificación de los animales que tuvo por objeto la comprension por parte del alumno de la clasificación categorizada de los animales, to que inplica un conocimiento previo que dará lugar, posteriormente, a una interpretación de los caminos seguidos por la evolución. La importancia del tema radica en que es fundamental comprender el ordenamiento lógico de los diferentes grupos de animales para llegar a asumir el significado de su anatomía y morfologia. Ha de tenerse siempre en cuenta que la clasificación animal debe ser natural $y$, en especial, filogenética; de esta forna, más adelante, se pueden comprender las relaciones de parentesco entre todos los grupos animales y, por ello, evitar errores de relación establecidos a base de carácteres no homologables o arbitrarios.

El estudio se llevó a cabo con alumnos de $6^{\circ}$ de EGB por considerarse que son los idóneos debido a su edad y a los conocimientos que ya poseen en el campo de la Zoologia. La experiencia constó de las siguientes fases:

1. Estudio del conocimiento que poseian los alumnos de enseñanza secundaria $\left(1^{\circ}\right.$ de BUP y $1^{\circ}$ de FP) sobre el tema: Se realizó por medio de encuestas que dieron a conocer el precario estado de los conocimientos filogenéticos por parte del alumnado.

2. Estudio de los conocimientos previos sobre el tema de los alumnos de $5^{\circ}$ de EGB: Se realizó también por medio de encuestas que nos dieron a conocer no sólo el estado de conocimientos sobre filogenia (prácticamente inexistentes) sino también los errores conceptales más frecuentes.

3. Trabajo con los alumnos de $6^{\circ}$ de EGB, que incluyó:

3.1. Fijación de conceptos válidos des- tinados a substituir los conceptos erróneos que los alumnos habian manifestado tener: Se lievó a cabo mediante observaciones, manipulaciones, representaciones e interpretación de información gráfica.

3.2. Estudio de tipos zoológicos fundamentales e intento de clasificación de los mismos por parte de los alumnos siguiendo unas premisas elaboradas por el profesor.

3.3. Comentario y explicación por parte del profesor de las bases en las que se asienta la Filogenia, explicando los conceptos fundamentales que se inciuyen en ellas.

Los resultados obtenidos pueden calificarse de buenos ya que, a pesar de la dificultad de comprensión de algunos conceptos, la mayoria de alumnos llegó a asimilarlos. De todas maneras hemos de hacer constar que la clase, en general, siempre tuvo muy buena predisposición hacia el tema y que las mayores dificultades fueron fruto de los errores conceptuales que poseían los alumnos, debidos en gran manera a la falta de formación de los maestros de cursos anteriores en esta materia y a la gran profusion de errores en conceptos filogenéticos que presentan la mayoría de libros de texto.

$\mathrm{X}$. Andrés,

Jefe del Dapartamento de Ciencias y Matemática, E.M.F.P.

«Narcís Monturiol», Barcelona.

J. Castelló

Departamento de Didáctica

de las Ciencias Experimentales y Matemáticas, Universidad de

Barcelona.

M.C. Sanz,

Departamento de Didáctica de las Ciencias Experimentales y Matemáticas, Universidad de Barcelona.

UN ESQUEMA CONCEPTUAL PARA LA ENSEÑANZA DE ESTEQUIOMETRÍA EN EL BACHILLERATO

Una de las dificultades que se presentan habitualmente en los primeros cursos de quimica de bachillerato es la enseñanza de los conceptos básicos de estequiometría.

Históricamente los profesores de cursos de química elemental, consideran que el concepto de «mol» es uno de los más dificiles de introducir y también de utilizar por sus alumnos en forma comprensiva.

Es habitual en los fibros de texto y también en los currículos de Bachilleratos la introducción del tema «estequiometriay en forma totalmente separada (un capítulo al comienzo o al final, según el autor) de los demás conceptos a desarrollar durante el curso de Química Elemental.

Esto hace que el alumno disocie totalmente los problemas propuestos, de las ecuaciones que están ralacionadas con ellos. Y además, cuando aprende a escribir las fórmulas de los diferentes compuestos y las ecuaciones quimicas correspondientes, no las relaciona con los cambios de masa que alli ocurren, sino que las considera simplemente como "paiabras" o esquemas verbales.

Es común que frente a una fórmula de un compuesto, por ejemplo: $\mathrm{CaCO}_{3}$, el alumno lea «cacotres», sin tener en cuenta lo que eso significa.

El esquema conceptual propuesto trata de superar esta disociación introduciendo los problemas estequiométricos en forma paulatina y paralela a los demás conceptos que se van desarroIlando.

El esquema propuesto es el de la figura I

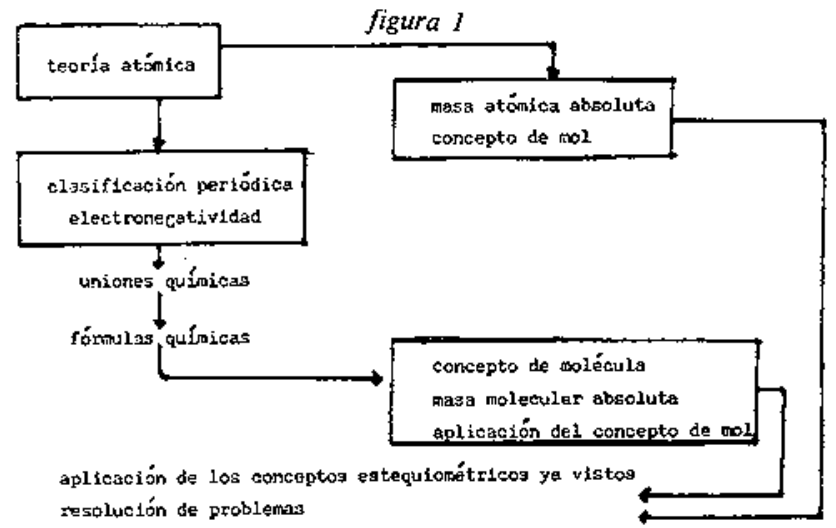


Puede deducirse el concepto de mol cuando el alumno conoce las masas de las partículas subatómicas. Conociendo el número atómico y el número másico puede calcular la masa atómica absoluta a partir de estos datos:

$$
\begin{gathered}
23 \\
\mathrm{Na} \\
11
\end{gathered}
$$

Puede deducirse fácilmente el número de protones y neutrones que posee el átomo de sodio. Estableciendo la relación entre la unidad de masa atónica y su equivalente en gramos:

$$
1 \mathrm{uma}=1.66 \times 10^{.24} \mathrm{~g}
$$

Si bien la equivalencia puede darse con más precisión, en cursos de bachillerato de los primetos años no es necesario una aproximación mayor, aunque desde luego esto no es objetable y queda a criterio del profesor utilizar mayor exactitud en la equivalencia.

Calculando el número de protones y neutrones se llegará a que el átomo de sodio tiene una masa absoluta de 23 umas. Luego:
1 uma

$1.66 \times 10^{-24} \mathrm{~g}$ $23 \mathrm{uma}-\longrightarrow-\cdots-3.818 \times 10^{-25} \mathrm{~g}$.

Puede sugerirse a los alumnos que averigüen el número de átomos de sodio presentes en $23 \mathrm{~g}$ de sodio, to que llevará a la siguiente conclusión:

$3,818 \times 10^{-25}$ g. - -1 átomo de $\mathrm{Na}$ 23 g. - - $6,02 \times 10^{23}$ átomos de $\mathrm{Na}$

Repitiendo este procedimiento con algunos ejemplos más, los alumnos 11 egan fácilmente a la definición de mol como «cantidad de materia que contiene $6,02 \times 10^{23}$ particulas", en este caso, átomos (1).

Cuando se conozca ya la formación de compuestos y los alumnos tengan la noción de molécula, se aplicará el procedimiento para llegar al mol de molécu. las y así el alumno estará capacitado para relacionar en cada caso la ecuación química con la correspondiente reiación estequiométrica, por ejemplo:

I mol de moléculas de oxigeno reacciona con 2 moles de moléculas de hidrogeno para dar 2 moles de moléculas de agua $\left(\mathrm{O}_{2}+2 \mathrm{H}_{2} \underset{2}{ } \underset{2}{2} \mathrm{H}_{2} \mathrm{O}\right)$.
De la misma mancra cuando escriba las ecuaciones correspondientes a la formación de óxidos, hidróxidos, ácidos y sales. De este modo, relacionando siempre las ecuaciones quimicas con sus correspondientes relaciones estequiométricas, se evita la disociación que ocurre habitualmente, y en cada caso es fácil hacer pequeños problemas relacionando masas y moles, etc.

Nota:

(1) Diversos autores comparten este criterio; asi se presenta en:

Staff or Research and Education Association, 1986, The Chemistry problem solver, (Research and Educa. tion Association: New York).

Bella, Alicia, 1984, Comunicación a la REQ 11. San Juan, 1984.

Beltrán, Faustino, 1980, Fórmulas quimicas ranozadas. (Plus Ulira: Buenos Aires).

Nella Bonetto de Fushimi Liceo Victor Mercante. UNLP. La Plata. Argentina.

\section{EXPERIENCIAS DE CLASE}

\section{ESTUDIO CINÉTICO DE UNA REACCIÓN DE COMBUSTIÓN}

La cinética química es uno de los temas presentes en el programa de la Quimica de COU y en el plan de estudios de Escuejas Universitarias de formación del Profesorado de EGB.

Nuestra experiencia docente nos ha hecho constatar, la dificuitad que encuentran los alumnos a la hora de adquirit y asimilar cada uno de los conceptos básicos relacionados con el tema.

Dada la conexión que existe entre la adquisición de conocimientos y las experiencias desarrolladas por los alumnos, creemos oportuno introducir la cinética quimica mediante una metodologia activa, a través de la cual, pondrán en práctica el método cientifico.

Para desarrollar los conceptos básicos implicados en el tema, tales como: relación concentración-tiempo, velocidad y orden de reacción, catálísis, etc; y con objeto de poner al estudiante en con* tacto con la construcción de gráficas e interpretación de las mismas, proponemos el estudio de una reacción de combustión.

Para esta reacción utilizamos comburentes adecuados, lo que permite controlar la velocidad de la reacción, haciéndola suficientemente lenta para que los alumnos puedan medir tiempos con facilidad con ayuda de un cronómetro.

Como comburente se utiliza nitrato de potasio y como combustible mechas de algodón del tipo $6 \mathrm{c}$.

Cada grupo de alumnos preparará 6 disoluciones de nitrato de potasio cuyas concentraciones varían desde $0.25 \mathrm{M}$ hasta disolución saturada, a la temperatura ambiente. A continuación sumergirán en cada disolución una mecha de hilo de algodón de $30 \mathrm{~cm}$. de longitud. El algodón se empapará de disolución, de modo que la cantidad de sustancia absorbida por unidad de longitud será proporcional a la concentra* ción de la disolución preparada.
Después se secará completamente el hilo de algodón con un secador de mano. Esta condición es imprescindible, ya que si el hilo está humedo, no habrá una combustión adecuada.

Se iniciará la combustión ascendentes de cada hilo de algodón acercando un ascua y se medirá con el cronómetro el tiempo que taróa en quemarse cada mecha.

Para la realización del estudio cinético los alumnos representarán las concentraciones (eje X) frente a los tiempos de combustion (eje Y) de las diferentes muestras. Se aprecia en dicha gráfica como a medida que aumenta la concentración de nitrato en el hilo, disminuye el tiempo de combustión ajustáncose la curva a una exponencial.

Si ahora se calculan los logaritmos de las concentraciones y se representan (eje $Y$ ), frente a los tiempos (eje X), se obtiene una línea recta de pendiente negativa, concluyéndose que la cinética es de primer orden, respecto a la concentración. 